\title{
Dinámica de raíces finas y su relación con la fertilidad edáfica en bosques pluviales tropicales del Chocó biogeográfico colombiano
}

\author{
Harley Quinto ${ }^{1 *}$, Haylin Caicedo ${ }^{1}$, May Thelis Perez $^{1} \&$ Flavio Moreno ${ }^{2}$ \\ 1. Programa de Biología. Facultad de Ciencias Básicas. Universidad Tecnológica del Chocó "Diego Luis Córdoba". 22 \\ Nº18B-10, Barrio Nicolás Medrano, Quibdó, Colombia. Código Postal 270002; hquintom@gmail.com \\ 2. Departamento de Ciencias Forestales. Facultad de Ciencias Agrarias. Universidad Nacional de Colombia Sede \\ Medellín. Código Postal 050034; fhmoreno@unal.edu.co \\ * Correspondencia
}

Recibido 18-XII-2015. Corregido 24-VI-2016. Aceptado 26-VII-2016.

\begin{abstract}
Fine root dynamics and its relationship with soil fertility in tropical rainforests of Chocó. The fine roots play an important role in the acquisition of water and minerals from the soil, the global carbon balance and mitigation of climate change. The dynamics (productivity and turnover) of fine roots is essential for nutrient cycling and carbon balance of forest ecosystems. The availability of soil water and nutrients has significantly determined the productivity and turnover of fine roots. It has been hypothesized that fine roots dynamics increases with the availability of soil resources in tropical forest ecosystems. To test this hypothesis in tropical rainforests of Chocó (ecosystems with the highest rainfall in the world), five one-ha permanent plots were established in the localities of Opogodó and Pacurita, where the productivity and turnover of fine roots were measured at $0-10 \mathrm{~cm}$ and $10-20 \mathrm{~cm}$ depth. The measurement of the fine root production was realized by the Ingrowth core method. The fine root turnover was measured like fine roots production divided mean annual biomass. In addition, soil fertility parameters $(\mathrm{pH}$, nutrients, and texture) were measured and their association with productivity and turnover of fine roots was evaluated. It was found that the sites had nutrient-poor soils. The localities also differ in soil; Opogodó has sandy soils and flat topography, and Pacurita has clay soils, rich in aluminum and mountainous topography. In Opogodó fine root production was $6.50 \pm 2.62 \mathrm{t} / \mathrm{ha}$.yr (mean $\pm \mathrm{SD})$. In Pacurita, fine root production was $3.61 \pm 0.88 \mathrm{t} / \mathrm{ha}$.yr. Also in Opogodó, the fine root turnover was higher than in Pacurita (1.17/y and $0.62 / \mathrm{y}$, respectively). Fine root turnover and production in the upper soil layers $(10 \mathrm{~cm}$ upper soil) was considerably higher. Productivity and turnover of fine roots showed positive correlation with $\mathrm{pH}$ and contents of organic matter, total $\mathrm{N}, \mathrm{K}, \mathrm{Mg}$, and sand; whereas correlations were negative with ECEC and contents of Al, silt, and clay. The percentage of sand was the parameter that best explained the variations of fine root production. The fine root turnover was negatively explained by soil Al availability. Results suggested the increase of fine root dynamics with soil fertility at a local scale, which also indicates that under the oligotrophic conditions of soils in tropical rainforests, fine roots tend to proliferate rapidly in small patches of soil rich in sand and nutrients. Rev. Biol. Trop. 64 (4): 1709-1719. Epub 2016 December 01.
\end{abstract}

Key words: carbon balance, nitrogen, Opogodó, Pacurita, permanent plots, net primary productivity, tropical soils.

La productividad de raíces finas (PRF) representa entre $30 \%$ y $40 \%$ de la productividad primaria neta (PPN) total de bosques tropicales (Jackson, Mooney, \& Schulze, 1997; Saugier, Roy, \& Mooney, 2001); además, juega un papel importante en el balance del carbono global y en la mitigación del cambio climático, en la dinámica subterránea del carbono, en la adquisición de agua y minerales del suelo y en el ciclo biogeoquímico de nutrientes (Vitousek \& Sanford, 1986; Silver et al., 2005). Sin embargo, a pesar de su importancia, la comprensión sobre la relación entre PPN y los factores del ambiente es limitada en bosques 
tropicales, pues en esta región son escasos los trabajos sobre PRF (Finér, Ohashi, Noguchi, \& Hirano, 2011).

El recambio de raíces finas (RRF) da cuenta del tiempo de crecimiento y renovación de las RF (Gill \& Jackson, 2000); en consecuencia, su estudio es fundamental en la comprensión de la dinámica del carbono subterráneo de bosques tropicales. Estudios recientes han mostrado que la PRF y el RRF en los trópicos están influenciadas por variables ambientales y biológicas. En el primer grupo están la latitud, temperatura, precipitación, humedad, aireación, nivel freático, acidez, textura y contenido de nutrientes del suelo (Gill \& Jackson, 2000; Silver et al., 2005; Metcalfe et al., 2008; Aragão et al., 2009; Finér et al., 2011). En el segundo grupo están la composición florística, edad del bosque, área basal, biomasa de raíces finas (BRF) y PPN aérea (Finér et al., 2011).

Diversos estudios se han realizado con el objetivo de explorar cómo los recursos edáficos controlan la dinámica y productividad de RF en bosques tropicales (Gill \& Jackson, 2000; Metcalfe et al., 2008; Kochsiek, Tan, \& Russo, 2013). Sin embargo, los resultados de tales estudios han sido variables y hasta contrastantes, por lo cual aún está por construirse una teoría que exprese la relación entre las propiedades del suelo y la dinámica de RF en bosques tropicales. Por ejemplo, Metcalfe et al., (2008), en bosques húmedos tropicales de la Amazonia, encontraron que la PRF incrementó en suelos con mayor contenido de humedad y nutrientes como N, P, Ca y Mg. Asimismo, Aragão et al., (2009) observaron un incremento en la PPN subterránea con el aumento en la disponibilidad de $\mathrm{P}$ edáfico en bosques de la Amazonia con suelos contrastantes. Al contrario, Kochsiek et al., (2013) reportaron un mayor crecimiento de RF en suelos arenosos, bien drenados y con menor contenido de nutrientes ( $\mathrm{P}, \mathrm{Ca}, \mathrm{Mg}$ y K ) en bosques tropicales de Malaysia; aunque, en ese mismo estudio se reportó una mayor PRF con el aumento en la tasa de suministro de iones $\mathrm{NH}_{4}{ }^{+}, \mathrm{PO}_{4}{ }^{3-}$ y $\mathrm{K}^{+}$(Kochsiek et al., 2013).

Las investigaciones de Metcalfe et al., (2008) y el meta-análisis realizado por Finér et al.,
(2011) reportan que la PRF y el RRF incrementan con el contenido de humedad y la precipitación, lo cual supone un posible cambio en la dinámica del carbono subterráneo con el incremento en las lluvias. Sin embargo, los estudios que evalúan la relación entre la dinámica de RF y las condiciones edáficas en bosques tropicales cubren rangos de precipitación inferiores a 5000 $\mathrm{mm}$ anuales (Gill \& Jackson, 2000; Metcalfe et al., 2008; Aragão et al., 2009; Kochsiek et al., 2013); y no se conocen estudios en bosques con precipitaciones mayores. Por lo tanto, se ignora cómo se relacionan la fertilidad edáfica con la PRF y RRF de bosques tropicales con alta precipitación (> $5000 \mathrm{~mm}$ anuales)? Nuestra hipótesis es que la PRF y RRF en bosques tropicales de alta pluviosidad aumentan con los contenidos de nutrientes del suelo. En tal sentido, el objetivo del presente trabajo fue evaluar la relación existente entre las condiciones edáficas y la dinámica de RF en bosques pluviales tropicales en el Chocó biogeográfico, donde algunos sitios presentan lluvias que superan los $10000 \mathrm{~mm}$ anuales (Poveda, Rojas, Rudas, \& Rangel, 2004), y que además, presentan suelos con topografías y texturas contrastantes (arenosas y arcillosas).

\section{MATERIALES Y MÉTODOS}

Área de estudio: El presente estudio se realizó en dos bosques pluviales tropicales: uno en la localidad de Pacurita (municipio de Quibdó) y otro en la localidad de Opogodó (municipio de Condoto), departamento del Chocó, Colombia. Estas dos localidades hacen parte de la subregión ecogeográfica Central Norte del Chocó biogeográfico, que comprende las cuencas altas de los ríos Atrato y San Juan (Poveda et al., 2004). Las localidades se encuentran dentro de la unidad geomorfológica de Colinas sedimentarias del Terciario, que se caracterizan por presentar bajas altitudes, están formadas por rocas sedimentarias, compuestas por arcillolitas arenosas, areniscas y calizas (West, 1957).

Ambos sitios presentan bosques pluviales tropicales y sus suelos son Ultisoles. 
Particularmente, la localidad de Opogodó, se encuentra ubicada a los $5^{\circ} 04^{\prime} 079^{\prime}$ "N-76 64 ' $47^{\prime}$ " $\mathrm{W}$ con una precipitación de $8000 \mathrm{~mm}$ anuales, a una altitud de 70 m.s.n.m, y una topografía plana. Los suelos son arenosos, muy ácidos, con baja capacidad de intercambio catiónico efectiva (CICE) y contenidos muy pobres de $\mathrm{P}, \mathrm{Mg}, \mathrm{y} \mathrm{Ca}$. Asimismo, las concentraciones de K son intermedias, y los valores de M.O. y $\mathrm{N}$ total son muy altos. Las especies arbóreas dominantes son Wettinia quinaria, Mabea occidentalis, Calophyllum auratum, Eschweilera sclerophylla, Oenocarpus bataua, entre otras (Quinto \& Moreno, 2016; Quinto, Moreno, Caicedo, \& Pérez, 2016). La localidad de Pacurita, se encuentra ubicada a los $5^{\circ} 41^{\prime} 55.8^{\prime \prime}$ N-76 35'59.4" W con una precipitación de 10 $000 \mathrm{~mm}$ anuales, con una altitud de entre 106130 m.s.n.m y una topografía montañosa. Los suelos son arcillosos y limosos, fuertemente ácidos, con porcentajes altos de saturación de $\mathrm{Al}$, y muy pobres de $\mathrm{P}, \mathrm{Mg}$, y Ca. Asimismo, la disponibilidad de K es intermedia, y la M.O. y $\mathrm{N}$ total son altas. Las especies dominantes son C. auratum, E. sclerophylla, O. bataua, Protium apiculatum, Brosimum utile, entre otras (Quinto \& Moreno, 2016; Quinto et al., 2016).

En la localidad de Opogodó, el muestreo se realizó en tres parcelas permanentes de una hectárea, instaladas en un bosque ubicado a cuatro kilómetros de la margen derecha de la carretera entre Opogodó y Novita, en predios de la Universidad Tecnológica del Chocó "Diego Luis Córdoba". En la localidad de Pacurita, el estudio se realizó en dos parcelas permanentes ubicadas en una zona de reserva forestal denominada Estación Biológica Pacurita, a $6.5 \mathrm{~km}$ del municipio de Quibdó, ubicada en la margen izquierda de la carretera entre Quibdó y Pacurita. En ambas localidades el muestreo fue realizado en bosques primarios bien conservados.

Establecimiento de parcelas: Se establecieron cinco parcelas permanentes en el año 2013; cada una consiste de un cuadrado de $100 \times 100 \mathrm{~m}$, dividido en 25 subparcelas de $20 \times 20 \mathrm{~m}\left(400 \mathrm{~m}^{2}\right)$; a su vez, las subparcelas se subdividieron en unidades de registro de 10 x $10 \mathrm{~m}$, en las cuales se tomaron las muestras de suelos y de BRF.

Medición de la productividad de raíces finas (PRF): Para la medición de la PRF se empleó una modificación del método de cilindros de crecimiento (ingrowth cores) (Cuevas \& Medina, 1988; Moreno, 2004). En la técnica original, se extrae un volumen de suelo conocido y se le retiran las raíces presentes; luego este volumen de suelo libre de raíces, se coloca en una malla cilíndrica, plástica o metálica, la cual se instala en el orificio dejado previamente en el piso del bosque. Una vez establecidos los cilindros de crecimiento, se dejan durante un período de tiempo, que permita el crecimiento de las raíces finas; luego se retiran a intervalos preestablecidos, se extraen las raíces y se determina la BRF y PRF (Cuevas \& Medina, 1988; Hendricks et al., 2006).

No obstante, con la técnica original de cilindros de crecimiento, algunos investigadores (Moreno, 2004) consideran que se presentan problemas prácticos para la recuperación de los cilindros de suelo una vez han crecido las raíces, lo cual genera incertidumbre del verdadero volumen de suelo evaluado y podría tener efectos de sobre o subestimación de la PRF. Por esta razón, en el presente estudio se empleó la modificación desarrollada por Moreno (2004), en la cual, en lugar de emplear cilindros o mallas metálicas para introducir el suelo libre de RF, se utilizaron tres alambres metálicos gruesos ubicados sobre las paredes del orificio dejado por el barreno. Estos alambres se usan para alinderar el espacio de llenado y como guía para el barreno durante la posterior recolección de las muestras. La ventaja de esta modificación es que se introduce el suelo en los hoyos hechos con barreno sin ningún empaque, de forma tal que el nuevo suelo está completamente en contacto con el medio circundante y se eliminan así los obstáculos que podrían limitar el crecimiento de las raíces. Asimismo, es importante mencionar que con este método se pueden recolectar pequeñas porciones de suelo intacto que no hacen parte del cilindro de suelo 
libre de raíces; de tal manera existe una leve incertidumbre en la precisión de este método.

Los cilindros de crecimiento modificados se ubicaron en el centro de cada cuadrícula de $10 \times 10 \mathrm{~m}$, y mediante barrenos Eijkelkamp ${ }^{\circledR}$ de suelos (de $8 \mathrm{~cm}$ de diámetro y $15 \mathrm{~cm}$ de profundidad) se extrajeron dos muestras a $0-10$ $\mathrm{cm}$ y $10-20 \mathrm{~cm}$ de profundidad. Posteriormente, se realizó una primera separación de las raíces finas $(\mathrm{RF})$ (con diámetros $\leq 5 \mathrm{~mm}$ ) en forma manual, empleando bandejas plásticas y tamices de diferente calibre $(0.5$ y $1.0 \mathrm{~mm})$. Luego de extraer las RF, el suelo fue introducido nuevamente en los orificios de los cuales fue extraído, mientras que las raíces fueron trasladadas al laboratorio de Botánica y Ecología de la Universidad Tecnológica del Chocó. Este procedimiento se realizó cada tres meses durante un año, y en cada muestreo se evaluaron 100 cilindros de suelo a dos profundidades por parcela, con lo cual se alcanzó un total de 1000 muestras de RF en cada muestreo en las cinco parcelas evaluadas.

En el laboratorio se hizo una última limpieza de las RF (para retirar partículas de suelo y hojarasca, principalmente) con tamices de diferentes calibres (de $0.5 \mathrm{~mm}$ y $1 \mathrm{~mm}$ de amplitud) y agua a presión. Posteriormente, la biomasa seca de RF se obtuvo mediante pesaje en balanza analítica (precisión $0.001 \mathrm{~g}$ ) después de secadas a $70^{\circ} \mathrm{C}$ en horno de ventilación forzada (Acequilab Ltda®) y se expresó en t/ha; la PRF se determinó como el valor acumulado a través del tiempo de la BRF en los cilindros de crecimiento y se expresó en t/ha.año.

\section{Medición del recambio de raíces finas} (RRF): Para su determinación se empleó la ecuación (1) (Aber, Melillo, Nadelhoffer, McClaugherty, \& Pastor, 1985; Aerts, Bakker, \& De Caluwe, 1992; Gill \& Jackson, 2000).

$$
\operatorname{RRF}\left(a \tilde{n} o^{-I}\right)=\mathrm{PRF} / \mathrm{BRF}
$$

Donde la PRF es la producción de raíces finas y BRF es la biomasa de raíces finas ("mean stading crop" en inglés). La ecuación (1) es una modificación del Modelo de Dahlman y Kucera (1965), en el cual una planta anual tendría un RRF de 1.0 año ${ }^{-1}$ si todas las raíces que produjo fueran a morir al final de la estación de crecimiento (Dahlman \& Kucera, 1965; Gill \& Jackson, 2000). El RRF se determinó para cada uno de los cilindros de crecimiento de las parcelas permanentes.

Para evaluar la relación entre la PRF y RRF con las propiedades del suelo; se emplearon los datos de textura, $\mathrm{pH}$, aluminio (Al) y contenido de nutrientes ( $\mathrm{P}, \mathrm{Ca}, \mathrm{K}, \mathrm{Mg}, \mathrm{CICE})$ de los suelos de las parcelas de Opogodó y Pacurita, que fueron publicados previamente por Quinto et al., (2016). Posteriormente, se evaluaron los supuestos de normalidad y homogeneidad de varianzas con los estadísticos de Bartlett, Hartley y Kurtosis (Hoshmand, 1998). Se empleó un análisis de componentes principales (ACP) para evaluar las relaciones lineales entre las variables edáficas, PRF y RRF. Puesto que algunas variables no cumplieron los supuestos de normalidad y homogeneidad de varianzas de los datos, la relación entre la dinámica de raíces (PRF y RRF) y las variables edáficas se evaluó mediante el Coeficiente de Correlación por Rangos de Spearman $\left(\mathrm{R}_{\mathrm{s}}\right)$, regresiones lineales y múltiples. La variación de PRF y RRF en función de las localidades y la profundidad del suelo se evaluó mediante la prueba no paramétrica de Mann-Whitney (W) (Hoshmand, 1998). Los análisis se realizaron con Statgraphics Centurion XV (Statistical Graphics Corp., 2002) y el entorno de programación R (R Core Team 2012).

\section{RESULTADOS}

Productividad y recambio de raíces finas: se presentaron diferencias significativas en los promedios de PRF ( $\mathrm{W}=-1614 ; \mathrm{P}<$ 0.0001 ) y de RRF ( $\mathrm{W}=-1$ 559; $\mathrm{P}<0.0001$ ) entre las localidades de Opogodó y Pacurita (Cuadro 1). En Opogodó se presentó una PRF (promedio $\pm \mathrm{DE}$ ) de $6.50 \pm 2.62 \mathrm{t} / \mathrm{ha}$.año, con un rango entre 3.50 y $19.81 \mathrm{t} /$ ha.año y un coeficiente de variación de 40.31 \%. En Pacurita la PRF fue menor, con promedio de $3.61 \pm 0.88$ t/ha.año, y rango entre 2.33 y 6.63 t/ha.año. Asimismo, en la localidad de Opogodó el RRF 
TABLE 1

Productivity and annual turnover of fine roots in two tropical rainforests of Choco, Colombia

CUADRO 1

Productividad y recambio anual de raíces finas en dos bosques pluviales tropicales del Chocó, Colombia

\begin{tabular}{|c|c|c|c|c|c|c|c|c|c|}
\hline \multicolumn{10}{|c|}{ Productividad de Raíces Finas (PRF) (t/ha.año) } \\
\hline Localidad & Profundidad $(\mathrm{cm})$ & PRF & D.E. & C.V. & E.E. & Min. & Max. & Kurtosis & Mann-Whitney \\
\hline Opogodó & $0-20$ & 6.50 & 2.62 & $40.31 \%$ & 0.30 & 3.50 & 19.81 & 8.95 & \multirow{2}{*}{$-1614 * * *$} \\
\hline Pacurita & $0-20$ & 3.61 & 0.88 & $24.35 \%$ & 0.12 & 2.33 & 6.63 & 1.71 & \\
\hline Opogodó & $0-10$ & 3.79 & 1.91 & $50.48 \%$ & 0.22 & 1.62 & 13.80 & 10.57 & \multirow{2}{*}{$-1446 * * *$} \\
\hline Pacurita & $0-10$ & 2.01 & 0.70 & $34.63 \%$ & 0.10 & 1.08 & 4.44 & 3.21 & \\
\hline Opogodó & $10-20$ & 2.71 & 1.10 & $40.72 \%$ & 0.13 & 1.39 & 6.95 & 3.81 & \multirow{2}{*}{$-1453 * * *$} \\
\hline Pacurita & $10-20$ & 1.60 & 0.42 & $26.58 \%$ & 0.06 & 0.95 & 2.61 & -0.35 & \\
\hline \multicolumn{10}{|c|}{ Recambio de Raíces Finas (RRF) (años ${ }^{-1}$ ) } \\
\hline Localidad & Profundidad $(\mathrm{cm})$ & RRF & D.E. & C.V. & E.E. & Min. & Max. & Kurtosis & Mann-Whitney \\
\hline Opogodó & $0-20$ & 1.17 & 0.55 & $46.79 \%$ & 0.06 & 0.55 & 4.28 & 13.69 & \multirow{2}{*}{$-1559 * * *$} \\
\hline Pacurita & $0-20$ & 0.62 & 0.26 & $41.21 \%$ & 0.04 & 0.23 & 1.76 & 7.31 & \\
\hline Opogodó & $0-10$ & 1.19 & 0.69 & $57.46 \%$ & 0.08 & 0.34 & 4.45 & 6.65 & \multirow{2}{*}{$-1290 * * *$} \\
\hline Pacurita & $0-10$ & 0.60 & 0.34 & $56.88 \%$ & 0.05 & 0.14 & 1.75 & 2.06 & \\
\hline Opogodó & $10-20$ & 1.23 & 0.48 & $38.78 \%$ & 0.05 & 0.70 & 3.95 & 14.14 & \multirow{2}{*}{$-1205^{* * *}$} \\
\hline Pacurita & $10-20$ & 0.77 & 0.33 & $43.08 \%$ & 0.05 & 0.32 & 1.78 & 0.36 & \\
\hline
\end{tabular}

D.E.: desviación estándar, C.V.: coeficiente de variación, E.E.: error estándar, Min: valor mínimo, Max: valor máximo.

fue mayor que en Pacurita (1.17 /año y 0.62 / año, respectivamente). Ambos sitios presentaron mayor PRF y RRF en los primeros $10 \mathrm{~cm}$ de profundidad del suelo (Cuadro 1).

Relación de las condiciones edáficas con la PRF y RRF: Las variables edáficas se alinearon a lo largo del primer eje del ACP; en particular, el contenido de arena, $\mathrm{pH}, \mathrm{MO}$ y $\mathrm{N}$ total. Los suelos de Opogodó presentaron mayor disponibilidad de estos elementos $y$, al mismo tiempo, mayor PRF y RRF, lo cual evidencia una mayor asociación entre dichas variables. Los primeros cuatro componentes del ACP fueron significativos (con valores propios mayores a 1); los dos primeros explicaron el $65 \%$ de la varianza total, mientras que los dos últimos explicaron solo el $16.5 \%$ (Fig. 1).

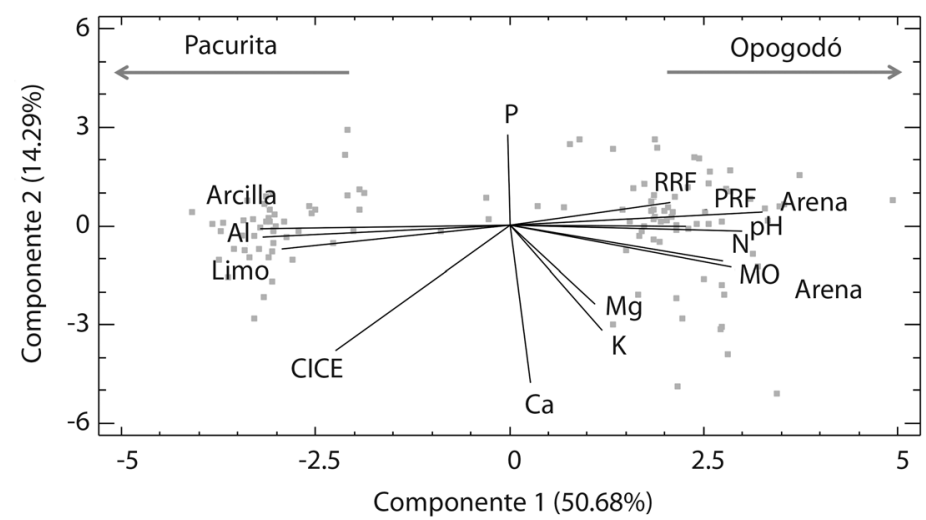

Fig. 1. Principal component analysis of soil variables, productivity and turnover of fine roots in two tropical rainforests of Choco, Colombia.

Fig. 1. Análisis de componentes principales de variables edáficas, productividad y recambio de raíces finas en dos bosques pluviales tropicales del Chocó, Colombia. 
El análisis de correlación mostró que la PRF y RRF se asociaron positivamente con $\mathrm{pH}, \mathrm{MO}, \mathrm{N}$ total, $\mathrm{K}, \mathrm{Mg}$ y arena. Mientras que, Al, CICE, limo y arcilla presentaron correlación negativa con la PRF y RRF (Cuadro 2). Mediante regresiones multiplicativas se evidenció que la dinámica de RF incrementó con el pH del suelo, el cual además explicó el $41.1 \%$ y $26.5 \%$ de la PRF y RRF, respectivamente (Fig. 2). El Al edáfico explicó el 52.3 $\%$ y el $44.6 \%$ de la variación en PRF y RRF, respectivamente; la dinámica de RF se redujo con el aumento en la disponibilidad de $\mathrm{Al}$ del suelo (Fig. 2). De otro lado, la relación entre la dinámica de RF y el contenido edáfico de MO y $\mathrm{N}$ total fue positiva; específicamente, la MO explicó $37.5 \%$ de la PRF y el $31.6 \%$ del RRF, mientras que el $\mathrm{N}$ total explicó $39.5 \%$ de la variación en PRF y el $34.8 \%$ del RRF (Fig. 3). La PRF presentó relación significativa, pero poco explicativa con el $\mathrm{K}\left(\mathrm{R}^{2}=10 \%\right.$;

TABLE 2

Spearman rank correlation coefficients among productivity and annual turnover of fine roots with soil parameters of two tropical rainforests of Chocó, Colombia

\section{CUADRO 2}

Coeficiente de correlación por rangos de Spearman de las tasas de productividad y recambio anual de raíces finas con los parámetros edáficos de dos bosques pluviales tropicales del Chocó biogeográfico, Colombia

\begin{tabular}{lcc}
$\quad \begin{array}{c}\text { Parámetros } \\
\text { edáficos }\end{array}$ & $\begin{array}{c}\text { Productividad de } \\
\text { Raíces Finas }\end{array}$ & $\begin{array}{c}\text { Recambio de } \\
\text { Raíces Finas }\end{array}$ \\
pH & $0.62 * * *$ & $0.51 * * *$ \\
Aluminio & $-0.68 * * *$ & $-0.67 * * *$ \\
Materia orgánica & $0.59 * * *$ & $0.55 * * *$ \\
Nitrógeno & $0.61 * * *$ & $0.57 * * *$ \\
Fósforo & $-0.11 \mathrm{~ns}$ & $-0.01 \mathrm{~ns}$ \\
Potasio & $0.35 * * *$ & $0.24 * *$ \\
Calcio & $0.02 \mathrm{~ns}$ & $-0.07 \mathrm{~ns}$ \\
Magnesio & $0.39 * * *$ & $0.35 * * *$ \\
CICE & $-0.48^{* * *}$ & $-0.51 * * *$ \\
Arena & $0.61 * * *$ & $0.54 * * *$ \\
Limo & $-0.57 * * *$ & $-0.52 * * *$ \\
Arcilla & $-0.66 * * *$ & $-0.56 * * *$ \\
\hline
\end{tabular}

1. Los asteriscos indican correlaciones significativas (*) $\mathrm{P}$ $<0.05 ;(* *) \mathrm{P}<0.01 ;(* *) \mathrm{P}<0.0001 ;$ ns $\mathrm{P}>0.05$.
$\mathrm{P}=0.0003), \operatorname{Mg}\left(\mathrm{R}^{2}=15 \% ; \mathrm{P}<0.0001\right) \mathrm{y}$ CICE $\left(\mathrm{R}^{2}=23 \%\right.$; $\left.\mathrm{P}<0.0001\right)$; asimismo, el RRF presentó relación significativa, pero poco explicativa con el K $\left(\mathrm{R}^{2}=10 \% ; \mathrm{P}=0.0003\right)$, $\mathrm{Mg}\left(\mathrm{R}^{2}=15 \% ; \mathrm{P}<0.0001\right)$ y $\operatorname{CICE}\left(\mathrm{R}^{2}=\right.$ $24 \% ; \mathrm{P}<0.0001)$ edáficos. El porcentaje de arena explicó el $41 \%$ y $34 \%$ de la variación en PRF y RRF, respectivamente. Por su parte, el limo explicó el 18\% y 15\% de la variación; mientras que, la arcilla explicó negativamente el $29 \%$ de la variación en PRF y un $20 \%$ del RRF. Los contenidos de $\mathrm{P}$ y Ca no presentaron relación significativa con la PRF y el RRF. Mediante regresiones lineales múltiples, se evidenció que las condiciones edáficas en conjunto explican un $52 \%$ de la variación en la PRF; siendo el contenido de arena, CICE, Limo, Mg, P y K, los parámetros que mejor explican tal variación. Por su parte, el RRF fue explicado principalmente por la disponibilidad de $\mathrm{Al}$ (Cuadro 3).

\section{DISCUSIÓN}

¿Qué tanto explica las condiciones edáficas los cambios en PRF y RRF en bosques tropicales con alta pluviosidad? En los bosques pluviales tropicales de Pacurita y Opogodó se presentaron tasas de PRF y RRF que incrementaron con el $\mathrm{pH}, \mathrm{MO}, \mathrm{N}$ total, $\mathrm{K}, \mathrm{Mg}, \mathrm{y}$ Arena. El aumento en la PRF y RRF con la disponibilidad de algunos nutrientes, a nivel local, ha sido reportado previamente por Metcalfe et al., (2008), quienes encontraron que la PRF incrementó con el contenido de humedad y nutrientes como N, P, Ca y Mg en bosques húmedos tropicales de la Amazonia. Igualmente, Kochsiek et al., (2013) reportaron un mayor crecimiento de RF en suelos con mayor tasa de suministro de iones $\mathrm{NH}_{4}^{+}, \mathrm{PO}_{4}^{3-}$ y $\mathrm{K}^{+}$en bosques de Malaysia. Estas tendencias en la relación PRF vs. nutrientes en suelos oligotróficos tropicales, probablemente se deben al hecho de que bajo condiciones de baja fertilidad edáfica las RF tienden a proliferar rápidamente en pequeñas áreas o parches del suelo más ricos en nutrientes (Robinson, Hodge, Griffiths, 

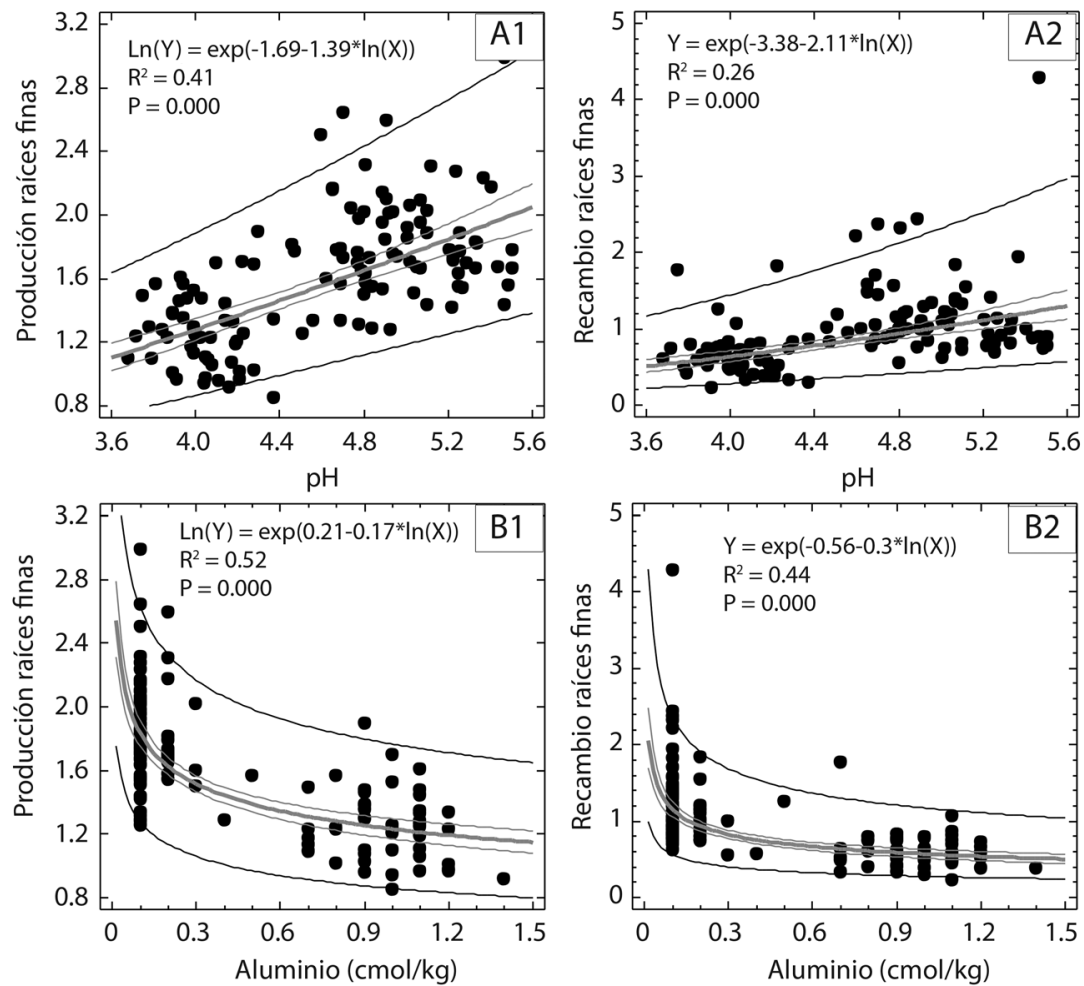

Fig. 2. Relationship between productivity (t/ha.yr) and turnover of fine roots (/yr) with soil $\mathrm{pH}$ and $\mathrm{Al}$ in tropical rainforests of Chocó, Colombia. Section A, Fine root dynamic and $\mathrm{pH}$. Section B, Fine root dynamic and Al.

Fig. 2. Relación entre la productividad ( $\mathrm{t} / \mathrm{ha} . \mathrm{año})$ y recambio de raíces finas (/año) con el pH y Al del suelo en bosques pluviales tropicales del Chocó biogeográfico, Colombia. Sección A. Dinámica de raíces finas y pH. Sección B: Dinámica de raíces finas y $\mathrm{Al}$.

\& Fitter, 1999; Kochsiek et al., 2013), como respuesta para reducir el déficit nutricional.

La relación positiva entre la PRF y nutrientes del suelo en los bosques pluviales del Chocó ofrece evidencia que apoya la hipótesis que plantea que a mayor disponibilidad en los recursos edáficos aumenta la dinámica de RF. Posiblemente, la PRF y RRF tienden a incrementar con la fertilidad del suelo en los bosques del Chocó, debido a la fuerte influencia de las lluvias sobre otras variables, como los nutrientes y humedad del suelo. $\mathrm{Al}$ respecto, se sabe que los altos niveles de pluviosidad provocan fuertes pérdidas de nutrientes por lixiviación; bajo estas circunstancias, los parches ricos en nutrientes parecen estimular el crecimiento y longevidad (tiempo de vida) de RF como estrategia que favorece la conservar de nutrientes dentro del sistema suelo-planta (Ryser 1996).

A pesar de que la relación entre la dinámica de RF y la disponibilidad de algunos nutrientes del suelo fue positiva en los bosques del Chocó, con el $\mathrm{P}$ y $\mathrm{Ca}$ edáficos, aisladamente, no hubo una asociación significativa. Probablemente, esta poca asociación se bebe a que la disponibilidad de estos nutrientes fue muy similar en las dos zonas evaluadas (rango de $\mathrm{P}=0.49-3.5 \mathrm{ppm}$ ) (Quinto et al., 2016), por lo que no se presentó un verdadero gradiente en su concentración. Contrario a esto, en las investigaciones realizadas por Aragão et al., (2009), se registró un incremento en la PPN subterránea con el aumento significativo 
TABLE 3

Multiple regressions of productivity rates and annual turnover of fine roots with soil parameters of two tropical rainforests of Chocó, Colombia

\section{CUADRO 3}

Regresión múltiple de las tasas de productividad y recambio anual de raíces finas con los parámetros edáficos de dos bosques pluviales tropicales del Chocó biogeográfico, Colombia

\begin{tabular}{|c|c|c|c|c|c|}
\hline \multicolumn{6}{|c|}{ Regresión múltiple de Ln(Producción de raíces finas) } \\
\hline Parámetros & Estimación & Error estándar & T estadística & P-valor & $\mathrm{R}^{2}$ ajustado \\
\hline Constante & -2.247 & 1.750 & -1.284 & 0.201 & 0.52 \\
\hline $\operatorname{Ln}(\mathrm{P})$ & -0.156 & 0.063 & -2.441 & 0.016 & \\
\hline $\operatorname{Ln}(\mathrm{Mg})$ & 0.222 & 0.078 & 2.818 & 0.005 & \\
\hline $\operatorname{Ln}(\mathrm{K})$ & 0.119 & 0.056 & 2.103 & 0.037 & \\
\hline CICE & -0.255 & 0.098 & -2.585 & 0.011 & \\
\hline $\operatorname{Ln}($ Arena $)$ & 0.954 & 0.308 & 3.090 & 0.002 & \\
\hline Ln(Limo) & 0.242 & 0.109 & 2.213 & 0.028 & \\
\hline
\end{tabular}

Análisis de varianza

\begin{tabular}{lccccc}
\multicolumn{1}{c}{ Fuente } & Suma de Cuadrados & G.L. & Promedio de Cuadrados & Prueba F & $P$-valor \\
Modelo & 10.375 & 6 & 1.729 & 21.07 & 0.0000 \\
Residuales & 9.358 & 114 & 0.082 & \\
Total (Corr.) & 19.733 & 120 & & \\
\multicolumn{7}{c}{ Regresión múltiple de Ln(Recambio de raíces finas) } \\
\multicolumn{7}{c}{ Parámetros } & Estimación & Error estándar & T estadística & P-valor & $\mathrm{R}^{2}$ ajustado \\
Constante & -0.558 & 0.0519 & -10.757 & 0.0000 & 0.43 \\
Ln(Al) & -0.3001 & 0.0312 & -9.619 & 0.0000 &
\end{tabular}

\begin{tabular}{lccccc}
\multicolumn{1}{r}{} & \multicolumn{2}{c}{ Análisis de varianza } \\
Fuente & Suma de Cuadrados & G.L. & Promedio de Cuadrados & Prueba F & $P$-valor \\
Modelo & 12.038 & 1 & 12.038 & 92.53 & 0.0000 \\
Residuales & 15.481 & 119 & 0.130 & & \\
Total (Corr.) & 27.52 & 120 & & \\
\hline
\end{tabular}

Backward was the method of selection of explanatory variables.

Backward fue el método de selección de variables explicativas.

en la disponibilidad de $\mathrm{P}$ edáfico (rango de $\mathrm{P}=$ 7.28-80.0 ppm) en bosques con suelos contrastantes de la Amazonia.

En los suelos de Opogodó, cuya textura es arenosa, se presentaron mayores tasas de PRF y RRF, similar a lo reportado por Metcalfe et al., (2008), Jiménez, Moreno, Peñuela, Patiño, y Lloyd, (2009) y Kochsiek et al., (2013). La alta PRF y RRF en suelos arenosos probablemente se debe a que con una mayor cantidad de arena en el suelo incrementa la presencia de macroporos, lo cual facilita el crecimiento de las RF. Asimismo, la textura arenosa presenta mayores tasas de liberación de nutrientes al suelo, en comparación con la textura arcillosa que tiende a retener más minerales como $\mathrm{NH}_{4}^{+}$, $\mathrm{PO}_{4}{ }^{3}, \mathrm{y} \mathrm{K}+$ (Kochsiek et al., 2013). Igualmente, la regresión múltiple evidenció que el porcentaje de arena, junto a otros parámetros (CICE, Limo, Mg, P, K), explica mejor que la MO y el $\mathrm{N}$ total, la variación en PRF. Por lo tanto, se puede concluir que en los bosques del Chocó la dinámica las RF está determinada, no solo por los nutrientes del suelo, sino también por la textura. Finalmente, es importante mencionar que la topografía de Opogodó puede que tenga una influencia significativa sobre la PRF; puesto que es más plana, lo cual reduce las pérdidas de nutrientes por escorrentía superficial y lixiviación. 

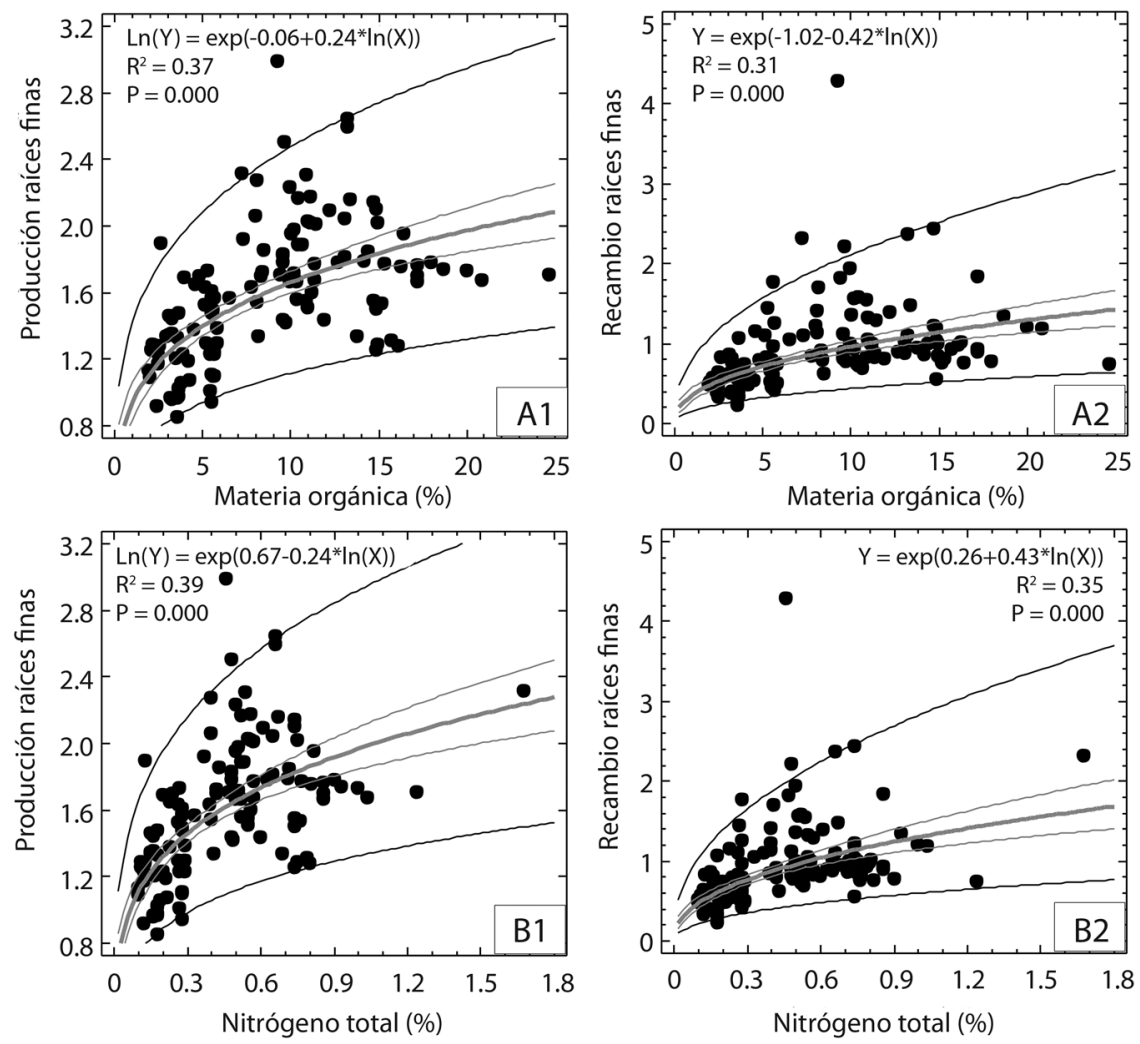

Fig. 3. Relationship between productivity (t/ha.yr) and turnover of fine roots (/yr) with soil organic matter and total $\mathrm{N}$ in tropical rainforests of Chocó, Colombia. Section A, Fine root dynamic and organic matter. Section B, Fine root dynamic and total $\mathrm{N}$.

Fig. 3. Relación entre la productividad (t/ha.año) y recambio de raíces finas (/año) con la materia orgánica y nitrógeno total del suelo en bosques pluviales tropicales del Chocó biogeográfico, Colombia. Sección A. Dinámica de raíces finas y materia orgánica. Sección B: Dinámica de raíces finas y Nitrógeno total.

La PRF y RRF se relacionan negativamente con Al, CICE, limo y arcilla. Particularmente, se observó que con altos contenidos de Al se redujo significativamente la PRF; esta reducción en PRF también fue reportada por Persson, Majdi, y Clemensson-Lindell, (1995) y Osaki, Watanabe, y Tadano, (1997), quienes consideraron que los altos niveles de saturación de $\mathrm{Al}$ en el suelo inhiben la elongación y penetración de las raíces, lo cual a su vez, reduce la absorción de agua y nutrientes. En una segunda etapa del daño, el Al obstaculiza la translocación de nutrientes a la parte aérea, lo cual se manifiesta como deficiencias nutricionales de $\mathrm{P}, \mathrm{Ca}$ y $\mathrm{Mg}$ principalmente (Sánchez \& Salinas 1983).

En conclusión, la dinámica de RF en los bosques pluviales tropicales del Chocó biogeográfico es muy sensible a las variaciones en las condiciones edáficas y topográficas. En tal sentido, se determinó que en las zonas con topografía plana, los incrementos edáficos en la disponibilidad de nutrientes y contenido de arena, al igual que una menor saturación de Al y acidez, tienden a facilitar el crecimiento y recambio de RF. Por lo tanto, en este estudio se corrobora la significativa influencia que tienen los suelos sobre la captura 
y dinámica del carbono en ecosistemas tropicales de alta precipitación.

\section{AGRADECIMIENTOS}

Esta investigación fue financiada por el Convenio 0010-2013 firmado entre la Universidad Tecnológica del Chocó "Diego Luis Córdoba", la Universidad Nacional de Colombia Sede Medellín y el Departamento Administrativo de Ciencia, Tecnología e Innovación - COLCIENCIAS, en el marco del proyecto titulado: "Evaluación del efecto de la fertilización del suelo sobre la productividad primaria neta de bosques pluviales tropicales del departamento del Chocó (CÓDIGO: 1128-569-35113)". Agradecemos la hospitalidad y colaboración de los habitantes de las localidades de Opogodó (municipio de Condoto) y Pacurita (municipio de Quibdó).

\section{RESUMEN}

Las raíces finas juegan un papel importante en la adquisición de agua y minerales del suelo, el balance global del carbono y la mitigación del cambio climático. La dinámica (productividad-PRF y recambio-RRF) de raíces finas es esencial para el ciclo de nutrientes y balance de carbono de los ecosistemas forestales. La disponibilidad de agua y nutrientes determina de manera significativa la PRF y el RRF. Se ha planteado la hipótesis de que la dinámica de raíces finas aumenta con la disponibilidad de los recursos del suelo en bosques tropicales. Para probar esta hipótesis en las selvas tropicales del Chocó (ecosistemas con las más altas tasas de precipitación en el mundo), se establecieron cinco parcelas permanentes de una hectárea en las localidades de Opogodó y Pacurita, donde la PRF y RRF se midieron a $0-10 \mathrm{~cm}$ y $10-20 \mathrm{~cm}$ de profundidad. La medición de la PRF se realizó por el método de cilindros de crecimiento. La tasa de RRF se midió como la PRF/ biomasa media anual. Además, se midieron los parámetros de fertilidad del suelo $(\mathrm{pH}$, nutrientes y textura) y se evaluó su asociación con PRF y RRF. Se encontró que ambos sitios presentan suelos pobres en nutrientes. Las localidades difieren en características edáficas; Opogodó tiene suelos arenosos y topografía plana, y Pacurita tiene suelos arcillosos, ricos en aluminio y topografía montañosa. En Opogodó la PRF fue (media \pm DE) de $6.50 \pm 2.62$ t/ha.año. En Pacurita la PRF fue $3.61 \pm 0.88 \mathrm{t} /$ ha.año. Igualmente, en Opogodó el RRF fue mayor que en Pacurita (1.17 /año y 0.62 /año, respectivamente). El RRF y la PRF fueron mayores en la superficie del suelo (10 $\mathrm{cm}$ de profundidad).
La PRF y el RRF mostraron correlación positiva con el $\mathrm{pH}, \mathrm{MO}, \mathrm{N}$ total, $\mathrm{K}, \mathrm{Mg}$, y arena; mientras que, las correlaciones fueron negativas con la $\mathrm{CICE}, \mathrm{Al}$, limo y arcilla. El porcentaje de arena fue el parámetro que mejor explica la variación en la PRF. La tasa negativa de la RRF fue explicada por la disponibilidad de Al. Los resultados evidenciaron un aumento en la dinámica de las raíces finas con la fertilidad del suelo a escala local, lo que sugiere que en selvas tropicales con suelos oligotróficos, las raíces finas tienden a proliferar rápidamente en pequeños parches de suelo ricos en arena y nutrientes.

Palabras clave: balance del carbono, nitrógeno, Opogodó, Pacurita, parcelas permanentes, productividad primaria neta, suelos tropicales.

\section{REFERENCIAS}

Aber, J. D., Melillo, J. M., Nadelhoffer, K. J., McClaugherty, C. A., \& Pastor, J. (1985). Fine root turnover in forest ecosystems in relation to quantity and form of nitrogen availability: a comparison of two methods. Oecologia, 66(3), 317-321.

Aerts, R., Bakker, C., \& De Caluwe, H. (1992). Root turnover as determinant of the cycling of $\mathrm{C}, \mathrm{N}$, and $\mathrm{P}$ in a dry heathland ecosystem. Biogeochemistry, 15(3), 175-190.

Aragão, L. E. O., Malhi, Y., Metcalfe, D. B., Silva-Espejo, J. E., Jiménez, E., Navarrete, D., Almeida, S., Costa, A. L., Salinas, N., Phillips, O. L., Anderson, L. O., Baker, T. R., Goncalvez, P. H., Huamán-Ovalle, J., Mamani-Solórzano, M., Meir, P., Monteagudo, A., Peñuela, M. C., Prieto, A., Quesada, C. A., RozasDávila, A., Rudas, A., Silva Junior, J. A., \& Vásquez, R. (2009). Above- and below-ground net primary productivity across ten Amazonian forests on contrasting soils. Biogeosciences, 6(12), 2759-2778.

Cuevas, E., \& Medina, E. (1988). Nutrient dynamics within Amazonian forests. II. Fine root growth, nutrient availability and leaf litter decomposition. Oecologia, $76(2), 222-235$.

Dahlman, R. C., \& Kucera, C. L. (1965). Root productivity and turnover in native prairie. Ecology, 46(1), 84-89.

Finér, L., Ohashi, M., Noguchi, K., \& Hirano, Y. (2011). Fine root production and turnover in forest ecosystems in relation to stand and environmental characteristics. Forest Ecology and Management, 262(11), 2008-2023.

Gill, R. A., \& Jackson, R. B. (2000). Global patterns of root turnover for terrestrial ecosystems. New Phytologist, 147(1), 13-31.

Hendricks, J. J., Hendrick, R. L., Wilson, C. A., Mitchell, R. J., Pecot, S. D., \& Guo, D. (2006). Assessing the patterns and controls of fine root dynamics: an 
empirical test and methodological review. Journal of Ecology, 94(1), 40-57.

Hoshmand, A. R. (1998). Statistical Methods for Environmental \& Agricultural Sciences. United States of America. Second edition. New York: CRC Press LLC.

Jackson, R. B., Mooney, H. A., \& Schulze, E. D. (1997). A global budget for fine root biomass, surface area, and nutrient contents. Proceedings of the National Academy of Sciences of the United States of America, 94(14), 7362-7366.

Jiménez, E., Moreno, F., Peñuela, M., Patiño, S., \& Lloyd, J. (2009). Fine root dynamics for forests on contrasting soils in the Colombian Amazon. Biogeosciences, 6 (12), 2809-2827.

Kochsiek, A., Tan, S., \& Russo, S. E. (2013). Fine root dynamics in relation to nutrients in oligotrophic Bornean rain forest soils. Plant Ecology, 214(5), 1385-0237.

Metcalfe, D. B., Meir, P., Aragao, L. E., Da Costa, A. C. L., Braga, A. P., Gonḉalves, P. H. L., De Athaydes Silva Jr., J., De Almeida, S. S., Dawson, L. A., Malhi, Y., \& Williams, M. (2008). The effects of water availability on root growth and morphology in an Amazon rainforest. Plant Soil, 311(1), 189-199.

Moreno-Hurtado, F. (2004). Soil Carbon Dynamics in Primary and Secondary Tropical Forests in Colombia (Doctoral thesis). Florida International University, Miami, Florida. USA.

Osaki, M., Watanabe, T., \& Tadano, T. (1997). Beneficial effect of aluminum on growth of plants adapted to low $\mathrm{pH}$ soils. Soil Science and Plant Nutrition, 43(3), 551-563.

Persson, H., Majdi, H., \& Clemensson-Lindell, A. (1995). Effects of acid deposition on tree roots. Ecology Bulletin, 44(1), 158-167.

Poveda, I. C., Rojas, C., Rudas, A., \& Rangel, O. (2004). El Chocó biogeográfico: Ambiente Físico. En O. Rangel (Ed.), Colombia Diversidad Biótica IV. El Chocó biogeográfico/ Costa Pacífica (pp. 1-21). Bogotá: Instituto de Ciencias Naturales. Universidad Nacional de Colombia.

Quinto, H., \& Moreno, F. (2016). Precipitation effects on soil characteristics in tropical rain forests of the Chocó biogeographical region. Revista Facultad Nacional de Agronomia, 69(1), 7813-7823.

Quinto, H., Moreno, F. H., Caicedo, M. H., \& Pérez, L. M. (2016). Biomasa de Raíces Finas y Fertilidad del Suelo en Bosques Pluviales Tropicales del Pacífico Colombiano. Colombia Forestal, 19(1), 53-66.

R Development Core Team. (2012). R: A language and environment for statistical computing. Vienna, Austria. R Foundation for Statistical Computing. ISBN: 3-900051-07-0. Recuperado de http://www.rproject.org/

Robinson, D., Hodge, A., Griffiths, B. S., \& Fitter, A. H. (1999). Plant root proliferation in nitrogen-rich patches confers competitive advantage. Proceedings of the Royal Society B: Biological Sciences, 266(1418), 431-435.

Ryser, P. (1996). The importance of tissue density for growth and life span of leaves and roots: a comparison of five ecologically contrasting grasses. Functional Ecology, 10(6), 717-723.

Sánchez, P. A., \& Salinas, J. G. (1983). Low input technology for managing Oxisol ans Ultisol in tropical America. Advances in Agronomy, 34(1), 279-406.

Saugier, B., Roy, J., \& Mooney, H. A. (2001). Estimations of global terrestrial productivity: Converging toward a single number? (pp 543-557). In J. Roy, B. Saugier, \& H. A. Mooney (Eds.), Terrestrial Global Productivity. San Diego: Academic Press.

Silver, W. L., Thompson, A. W., Mcgroddy, M. E., Varner, R. K., Dias, J. D., Silva, H., Crill, P. M., \& Keller, M. (2005). Fine root dynamics and trace gas fluxes in two lowland tropical forest soils. Global Change Biology, 11(2), 290-306.

Statistical Graphics Corp. 2002.StatgraphicsPlusCenturium Version 5.1. Descargado de www.Statgraphics.Com.

Vitousek, P. M., \& Sanford, R. L. (1986). Nutrient cycling in moist tropical forest. Annual Review of Ecology and Systematics, 17, 137-167.

West, R. (1957). Las tierras bajas del Pacífico colombiano. Bogotá DC. Instituto Colombiano de Antropología: Imprenta Nacional de Colombia. 
